\title{
Identification and Active Feedback-Feedforward Control of Rotor
}

\author{
Kari M. J. Tammi \\ VTT - Technical Research Centre of Finland, P.O. Box 1000, FI-02044 VTT, Finland
}

(Received 3 October 2005; revised 13 February 2006; accepted 26 January 2007)

\begin{abstract}
This experimental work presented demonstrates the use of identification, feedback, and feedforward methods to control rotor vibrations. The experiments were performed on a rotor test rig having a 3-kg rotor supported by journal bearings; the first bending resonance of the rotor shaft was about $50 \mathrm{~Hz}$. Identification was carried out with a method taking into account the disturbances due to rotation. The method, using a reference signal generated from speed measurement, was able to discard the forced vibrations due to the mass imbalance. The active control objective was to reduce the radial response at the rotor midpoint by an electromagnetic actuator located outside the bearing span of the rotor. The feedback system was a proportional-derivative-type controller, which increased the damping of the system. A feedforward control system was constructed to work together with the feedback controller. The control methods produced a significant decrease in the midpoint responses of the rotor at sub-critical speeds. For supercritical speeds, the decrease in the responses was more modest due to the restricted control authority. The stability of the feedforward controller was studied in order to explore the relationship between the system damping and the modelling accuracy required by the feedforward control system.
\end{abstract}

\section{INTRODUCTION}

This research was carried out in order to study the use of a supplementary actuator for rotor vibration control. The general aim was to explore vibration control solutions for rotor systems with conventional bearings, not to carry the rotor mass by the actuator. The objective of this study was to control the vibrations of the rotor in the test rig by using an electromagnetic actuator. The vibrations were to be attenuated inside the bearing span of the rotor while the actuator was located outside the bearing span. The aim was also to identify the dynamic rotor system by using the data acquired during its operation. Even the test environment used was relatively small; the intention was to expand the principle to larger prototypes and applications to control resonance vibrations of heavy rotors with a supplementary actuator. Research on similar set-ups has been carried out by Cheung et al., ${ }^{1}$ and Ishimatsu et al. ${ }^{2}$

The test environment had a $560 \mathrm{~mm}$ long slim shaft with three disks attached (Figs. 1 and 2). The diameter of the shaft was $10 \mathrm{~mm}$. The total weight of the rotor including the shaft and the disks was $2.7 \mathrm{~kg}$. The rotor was supported by journal bearings whose mutual distance was $360 \mathrm{~mm}$. The rotor was driven by an electrical motor by means of a flexible coupling. Radial displacement sensors were placed at two locations along the shaft: at the midpoint and at the end of the rotor (S1 and S2 in Fig. 1). The control system design consisted of two topologies: feedback control to provide basic damping over a wider frequency band, and feedforward control to compensate the disturbance due to the rotor mass unbalance. The feedforward control algorithm used the midpoint displacement sensors and the feedback algorithm used the endpoint displacement sensors to provide the control error signal. The speed of rotation was measured with a pulse sensor at the drive end of the rotor. The rotation speed measurement was used for generating the reference signal for identification and feedforward control. Also, the speed measurement was used by the feedforward controller in order to estimate the system frequency response at the speed of rotation. The journal bearings, the actuator, the displacement sensors, and the driving motor were all fixed to a stiff foundation considered ideally rigid in this study.

The electromagnetic actuator was used to produce the control forces at the non-drive end of the rotor. The actuator and its control unit were modified from active magnetic bearing equipment. Being a non-contact device, the actuator exerted the control forces through an air gap on the armature fixed to the rotor (Disk 3 in Fig. 1). The control unit had its own internal force control loop to make the force generation more accurate. The forces exerted were controlled by means of feedback from the magnetic flux density sensors in the air gap between the rotor and the actuator. This control loop was not subjected to any research in this work. The actuator, the control unit hardware, and its internal force control loop were assumed to be "sufficiently fast" for the purpose. The design specification of the actuator was to be capable to produce a force of $500 \mathrm{~N}$ up to $500 \mathrm{~Hz}$, whereas the control action took place in the frequency band from 0 to $150 \mathrm{~Hz}$ and only a few newtons were required. The forces used were low, in the order of one third of the rotor weight. Thus, the actuator was oversized for this particular purpose. This was due to technical limitations in scaling the system down and to the availability of ready-made magnetic bearing technology. The components of the set-up and their modifications from the original purposes have been reported in more detail by Tammi. ${ }^{3}$ In contrast to magnetic bearings, the objective was not to have the actuator carry static loads, as the rotor was supported by the journal bearings.

The main contributions of this paper are 1) to present an identification method that automatically discards disturbances correlated with a reference signal, and 2) to demonstrate the roles of feedback and feedforward control algorithms in rotor vibration control. The identification method is 